\title{
LMS\&BRLS Interference Suppression for Spread Communications
}

\author{
Xin-Quan Luo
}

Department of science and Engineering, Communication University of China, Beijing,100024,China

xqluo@cuc.edu.cn

Keywords:DS-CDMA,narrow-band interference suppression,multiple-access interference suppression, blind adaptive LMS \& RLS algorithm

Abstract. A LMS predictive \& Blind RLS code-aided technique for simultaneous suppression of multiple-access interference (MAI) and AR narrow-band interference (NBI) in direct sequence-code division multiple access (DS-CDMA) systems is proposed. This technique is based on combining predictive and code-aided approach, and the proposed AR suppression technique can beimplemen tedin a blind adaptive mode. The convergence condition and the steady state performance of it are analyzed. Simulation proves its superiority than adaptive BRLS technique.

\section{Introduction}

Over the past years, the valid time domain MAI and NBI suppression methods used in DSCAMA systems can be effectively grouped into predictive and code-aided techniques. For predictive techniques ${ }^{[1-4]}$, they include linear predictive methods that make use of DS-CDMA and NBI's spectral properties and nonlinear predictive methods that make use of these signals'spectra and firstorder probability distribution. These studies focused on a receiver structure in which NBI is handled through a dedicated suppression block, whereas MAI suppression is performed through conventional matched filters. The conclusion is that the effectiveness of these systems in NBI suppression decreased for an increasing number of the users ${ }^{[5-6]}$. For code-aided techniques ${ }^{[7-9]}$, they make use of the spreading codes of the interest signal and the second-order statistics of NBI. Code-aided techniques detect the interest user by batch processing of the observables and achieve simultaneously rejecting MAI and NBI. Overall, code-aided techniques are clearly superior to predictive techniques.

However, existing code-aided technique could only effectively suppress sinusoidal signal, digital signal and autoregressive (AR) which spectral density is exceeding sharply peaked. In order to improve the performance of code-aided techniques when the AR has a mild sharply peak, a LMS predictive \& Blind RLS code-aided technique is proposed with the idea of combining predictive and code-aided approach ${ }^{[10-11]}$. The convergence condition and the steady state performance of it are analyzed. Simulation proves its superiority than adaptive BRLS technique.

\section{Signal Model}

Consider a baseband synchronous DS-CDMA network of $K$ users. The received discrete-time sequence by chip pulses can be modeled as

$$
\begin{aligned}
r(m) & =y(m)+i(m)+\varepsilon(m) \\
& =\sum_{k=1}^{K} A_{k} \sum_{n=-\infty}^{\infty} b_{k}(n) s_{k}(m-n N)+i(m)+\varepsilon(m)
\end{aligned}
$$

Where $y(m)$ is the DS-CDMA siganl, $i(m)$ is the NBI, $\varepsilon(m)$ is the white Gaussian noise, $N$ is processing Gain, and $A_{k}, b_{k}(n), s_{k}(m)$ representthe received amplitude, symbol stream and normalized signaling waveform of the $k$ th user.

As a consequence, global second-order measures such as covariance sequence and covariance matrix are of primary concern.Strictly speaking, the covariance properties of the received signal should be given through

$$
\begin{aligned}
\rho_{r r}(m) & =\rho_{y y}(m)+\rho_{i i}(m)+\rho_{\varepsilon \varepsilon}(m) \\
& =\sum_{k=1}^{K} \sigma_{y, k}^{2} \delta(m)+\sigma_{i}^{2} \alpha^{|m|}+\sigma_{\varepsilon}^{2} \delta(m)
\end{aligned}
$$




$$
\begin{aligned}
\boldsymbol{R}_{r r}(n) & =\boldsymbol{R}_{y y}(n)+\boldsymbol{R}_{i i}(n)+\boldsymbol{R}_{\varepsilon \varepsilon}(n) \\
& =\sum_{k=1}^{K} A_{k}^{2} \boldsymbol{s}_{k} \boldsymbol{s}_{k}^{\mathrm{T}}+\boldsymbol{R}_{i i}(n)+\sigma_{\varepsilon}^{2} \boldsymbol{I}_{N}
\end{aligned}
$$

Where $\rho_{y y}(m), \rho_{i i}(m), \rho_{\varepsilon \varepsilon}(m)$ and $\boldsymbol{R}_{y y}(n), \boldsymbol{R}_{i i}(n), \boldsymbol{R}_{\varepsilon \varepsilon}(n)$ denote, respectively, the covariance sequence and the covariance matrix of DS-CDMA signal, NBI and white Gaussian noise.Here, $\sigma_{y, k}^{2}$, $\sigma_{i}^{2}, \sigma_{\varepsilon}^{2}$ present the covariance of DS-CDMA signal, NBI and white Gaussian noise respectively. $\alpha \in(0,1)$ denotes the sharp of one order AR's spectral density.As $\alpha \rightarrow 1$, the spectral density becomes more sharply peaked. $s_{k}=\left[s_{k, N-1}, s_{k, N-2}, \cdots, s_{k, 0}\right]^{\mathrm{T}} / \sqrt{N}$ is the vector arising from projection the normalized signaling waveform of the $k$ th user. Besides, $\boldsymbol{R}_{i i}(n)$ defined as

$$
\boldsymbol{R}_{i i}=\sigma_{i}^{2}\left[\begin{array}{cccc}
1 & \alpha & \cdots & \alpha^{N-1} \\
\vdots & \ddots & \vdots \\
\alpha^{N-1} & \alpha^{N-2} & \cdots & 1
\end{array}\right]
$$

\section{Blind Adaptive LMS Predictive \& RLS Code-Aided Implementation}

\section{Adaptive LMS \& BRLS Detector}

This paper only consider the case that User 1 is the interest user and others are MAI. As a useful measure of performance, the optimal SINR of code-aided detector has been considered previously ${ }^{[8-}$ ${ }^{9,12]}$. Such as

$$
\operatorname{SINR}=N \sigma_{y, 1}^{2} \mathbf{s}_{1}^{\mathrm{T}}\left(\sum_{k=2}^{K} A_{k}^{2} \boldsymbol{s}_{k} \boldsymbol{s}_{k}^{\mathrm{T}}+\boldsymbol{R}_{i i}+\sigma_{\varepsilon}^{2} \boldsymbol{I}_{N}\right)^{-1} \boldsymbol{s}_{1}
$$

The method to increase the optimal SINR of code-aided detector can be given as

1) Increase $N \sigma_{y, 1}^{2}$, which equal to increase the power of interest user. It couldn't be adopted because it will aggravate the burden of sending set.

2) Decrease $A_{k}^{2}, k \in(2, K)$, which equal to decrease the power of MAI. It couldn't be adopted too because the other users can't be control easily.

3) Decrease $\sigma_{\varepsilon}^{2}$, which equal to decrease the power of ambient channel noise. It also couldn't be adopted because the $\sigma_{\varepsilon}^{2}$ which relative to interest user is generally low.

4) Decrease the power of $\boldsymbol{R}_{i i}$, which equal to decrease a partial power of NBI before optimal code-aided detector. For this purpose, it needs a method that could decrease the power of NBI, make no difference of DS-CDMA signal and have a low complexity. Fortunately, linear prediction is an apropos technique to satisfy the conditions above. Besides that, it could realize adaptive detection and blind detection easily.

So, the method of adding prediction could well be adopted. This paper proposed the LMS linear prediction and Blind RLS (BRLS) code-aided techniques.Fig.1 shows the block diagram of the LMS predictive \& BRLS code-aided technique. To implement a blind adaptive arithmetic, the stepsize $\mu$, vector $\boldsymbol{q}(m)=\left[q_{1}(m), q_{2}(m), \cdots q_{M}(m)\right]^{\mathrm{T}}$ of prediction and forgetting factor $\lambda$, vector $\boldsymbol{w}_{1}(n)=\left[\boldsymbol{w}_{11}(n), \boldsymbol{w}_{12}(n), \cdots \boldsymbol{w}_{1 N}(n)\right]^{\mathrm{T}}$ of code-aided are used.

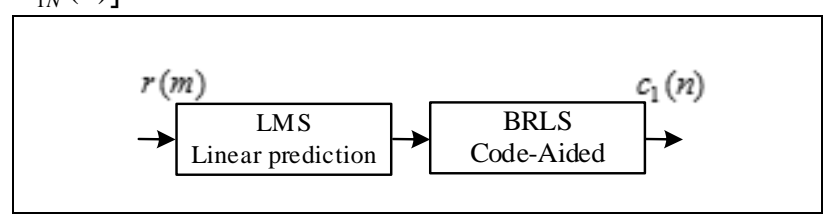

Figure.1. Block diagram of the LMS predictive \&BRLS code-aided technique

\section{Adaptive LMS Predictive Filter}

The resulted signal which subtracting the NBI estimate is

$$
\tilde{r}(m)=r(m)-\boldsymbol{q}^{\mathrm{T}}(m-1) \boldsymbol{r}_{M}(m)
$$

Where $\boldsymbol{r}_{M}(m)=[r(m-1), r(m-2), \cdots r(m-M)]^{\mathrm{T}}$. 
The update equation of LMS algorithm is ${ }^{[10-11]}$

$$
\boldsymbol{q}(m)=\boldsymbol{q}(m-1)+2 \mu \tilde{r}(m) \boldsymbol{r}_{M}(m)
$$

And the predictive matrix is defined as

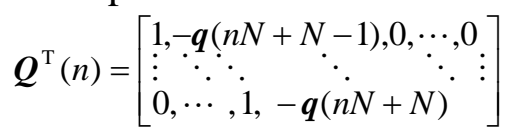

\section{BRLS Code-Aided Filter}

Afterwindowing the adaptive LMS filter signal $\tilde{\boldsymbol{r}}(n)=[\tilde{r}(n N+N-1), \tilde{r}(n N+N-2), \cdots \tilde{r}(n N)]^{T}$ and using the rule of minimum mean output energy (MMOE), the update equation of BRLS algorithm is ${ }^{[8-9]}$

$$
\begin{aligned}
\boldsymbol{k}(n) & =\frac{\boldsymbol{C}(n-1) \tilde{\boldsymbol{r}}(n)}{\lambda+\tilde{\boldsymbol{r}}^{\mathrm{T}}(n) \boldsymbol{C}(n-1) \tilde{\boldsymbol{r}}(n)} \\
\tilde{\boldsymbol{C}}(n) & =\tilde{\lambda}^{-1}\left[\tilde{\boldsymbol{C}}(n-1)-\tilde{\boldsymbol{k}}(n) \boldsymbol{r}^{\mathrm{T}}(n) \tilde{\boldsymbol{C}}(n-1)\right] \\
\boldsymbol{w}_{1}(n) & =\frac{\tilde{\boldsymbol{C}}(n) \boldsymbol{s}_{11}(n)}{\boldsymbol{s}_{11}^{\mathrm{T}}(n) \tilde{\boldsymbol{C}}(n) \boldsymbol{s}_{11}(n)}
\end{aligned}
$$

Here, especially, we modify the interest signature vector as

$$
\boldsymbol{s}_{11}(n)=\boldsymbol{Q}^{\mathrm{T}}(n)\left[\begin{array}{ll}
\boldsymbol{s}_{1}^{\mathrm{T}} & \boldsymbol{s}_{1(1: M)}^{\mathrm{T}}
\end{array}\right]^{\mathrm{T}}
$$

\section{Convergence Analysis and Steady State Misadjustment}

1) Convergence condition of the mean tap weight vector

$$
\begin{aligned}
& 0<2 \mu<<1 / \operatorname{tr}\left\{\boldsymbol{R}_{r_{M} r_{M}}\right\} \\
& 0<\lambda<1
\end{aligned}
$$

2) Malajustment in MSE

$$
\begin{aligned}
& \delta_{L M S}=\mu \operatorname{tr}\left\{\boldsymbol{R}_{r_{M} r_{M}}\right\} \\
& \delta_{\text {BRLS }}=\frac{1-\lambda}{2 \lambda}(N-1)
\end{aligned}
$$

3) Steady-state SINR

$$
S I N R_{L M S \& B R L S}=\frac{\operatorname{SINR}_{C A}^{o}}{1+\delta_{B R L S}+\delta_{B R L S} S I N R_{C A}^{o}}
$$

Where

$$
\operatorname{SINR}_{C A}^{o}=A_{1}^{2} \boldsymbol{s}_{1}^{\mathrm{T}}\left(\sum_{k=2}^{K} A_{k}^{2} \boldsymbol{s}_{k} \boldsymbol{s}_{k}^{\mathrm{T}}+\frac{\tilde{\sigma}_{i}^{2}\left(1+\delta_{L M S}\right)}{\sigma_{i}^{2}} \boldsymbol{R}_{i i}+\sigma_{\varepsilon}^{2} \boldsymbol{I}_{N}\right)^{-1} \boldsymbol{s}_{1}
$$

\section{Simulations}

Fig.1gives the comparison of the MSE performance by adaptive LMS \& BRLS and adaptive BRLS arithmetic.The NBI is a one-order AR with $\alpha=-0.95$. The white noise power is held constant at-20dB, while each power ofthree MAI is $10 \mathrm{~dB}$ higher that user 1 and the NBIpower is 20dB.The data plotted are the average over 500 simulations with $\mu=0.0005$ and $\lambda=0.999$.

It is seen from the Fig 2 that the MSE performance of adaptive LMS \& BRLSalgorithm is notability superior to adaptive BRLS algorithm. 


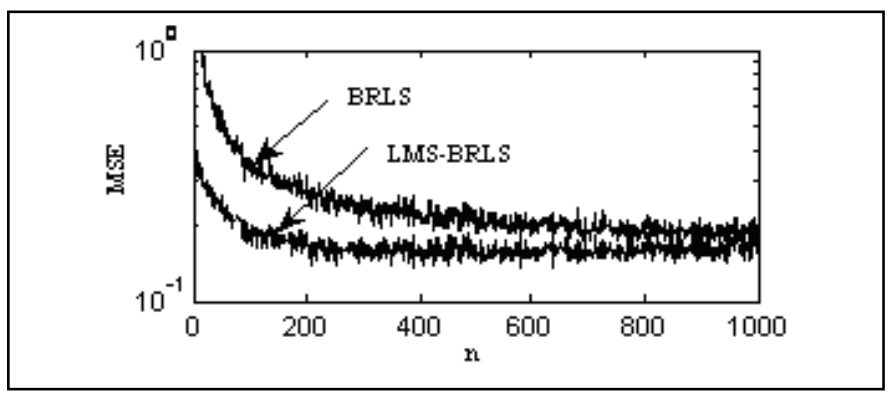

Figure.2.Comparison of the MSE performance by adaptive LMS \& BRLS and adaptive BRLS

Fig 3gives the tracking behavior of LMS \& BRLS algorithm. The simulation starts with one desired user's signal, three MAI signal with each of $10 \mathrm{~dB}$ and a strong one order AR with $20 \mathrm{~dB}$. At timen=500, another three MAI signal with each of $10 \mathrm{~dB}$ added. At time $n=1000$,three of the original MAI are removed from the system.

Fig 3illustrates the tracking behavior of the adaptive LMS \& BRLS in a mobile environment which existing NBI.

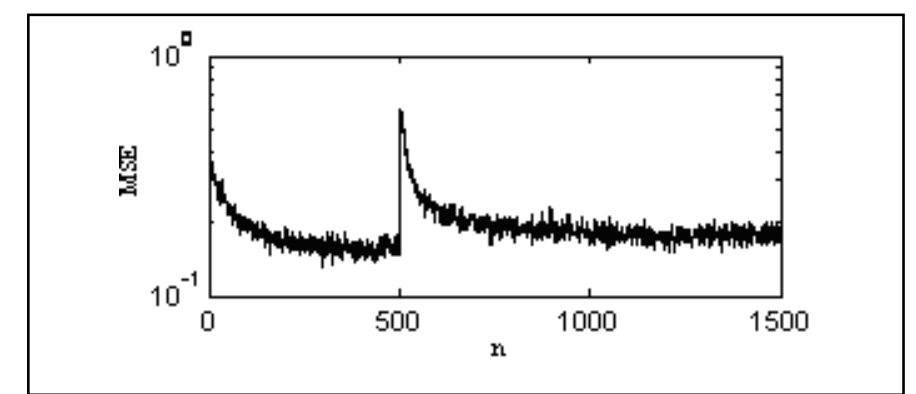

Figure.3.Tracking behavior of LMS \& BRLS algorithm

\section{Summary}

In this paper, a predictive \& code-aided technique for simultaneous suppressing MAI and NBI in DS-CDMA systemsis considered. This technique is based on combining predictive and code-aided approach,developed and analyzeda adaptive LMS \&BRLSversion of this interference suppression algorithm. Simulation proves its superiority than adaptive BRLS technique.

\section{References}

[1] ELIAS M. Closed-form analytical results for the rejection of narrow-band interference in PN spread-spectrum systems-Part I: Linear prediction filters[J]. IEEE Trans Commun, 1984,32(8):888896.

[2] ELIAS M. Closed-form analytical results for the rejection of narrow-band interference in PN spread-spectrum systems-Part II: Linear interpolation filters[J]. IEEE Trans Commun, 1985,33(1):10-19.

[3] WANG Kun-jie, ZHOU Zu-cheng, YAO Yan. Closed-form analytical results for interference rejection of nonlinear prediction filters in DS-SS systems[J]. Electron lett, 1997,33(16):1354-1355.

[4] WANG Kun-jie, ZHOU Zu-cheng, YAO Yan.Performance analysis of nonlinear interpolation filters in DS spread spectrum systems under narrowband interference conditon[J]. Electronics letters, 1998,34(15):1464-1465.

[5] POOR H V, RUSCH L A. Narrowband interference suppression in spread spectrum CDMA[J]. IEEE Pers Commun. 1994,1(3):14-27.

[6] POOR H V. Active interference suppression in CDMA overlay systems[J]. IEEE J Sel Areas Commun, 2001,19(1):4-20. 
[7] POOR H V, WANG Xiaodong. Code-aided interference suppression for DS/CDMA communications-Part I: Interference suppression capability[J]. IEEE Trans Commun, 1997, 45(9):1101-1111.

[8] POOR H V, WANG Xiaodong. Code-aided interference suppression in DS/CDMA communications-II: Parallel blind adaptive implementations[J]. IEEE Trans Commun, 1997,45(9):1112-1122.

[9] BUZZI S, LOPS M, POOR H V. Code-aided interference suppression for DS/CDMA overlay systems[J]. Proc IEEE, 2002,90(3):394-435.

[10] LU Xiao-ning, HO K C. Novel adaptive methods for narrowband interference cancellation in CDMA multi-user detection[A]. 2005 IEEE International Conference on Acoustics, Speech, and Signal Processing[C].United States:Institute of Electrical and Electronics Engineers Inc, 2005. 1-4.

[11] HO K C, LU Xiao-ning, MEHTA Vandana. Adaptive Blind narrowband interference cancellation for multi-user detection[J]. IEEE Trans Wireless Commun. 2007,6(3):1024-1033.

[12] LI Lin, ZHOU Wen-hui, TAN Shu-sen. Optimal interference suppression technique in the MBER sense for spread spectrum systems[J]. Acta Electronica Sinica, 2005,33(1):32-37. 